\title{
Diameter dependence of the defect-induced Raman modes in functionalized carbon nanotubes
}

\author{
Jan Laudenbach ${ }^{\mathrm{a}}$, Daniel Schmid ${ }^{\mathrm{a}}$, Felix Herziger ${ }^{\mathrm{a}}$, Frank Hennrich ${ }^{\mathrm{b}}$, \\ Manfred Kappes ${ }^{\mathrm{b}}$, Matthias Muoth ${ }^{\mathrm{c}}$, Miroslav Haluska ${ }^{\mathrm{c}}$, Ferdinand Hof ${ }^{\mathrm{d}}$, \\ Claudia Backes ${ }^{\mathrm{e}, \mathrm{f}}$, Frank Hauke ${ }^{\mathrm{f}}$, Andreas Hirsch ${ }^{\mathrm{f}, \mathrm{g}}$, Janina Maultzsch ${ }^{\mathrm{a}, *}$ \\ ${ }^{a}$ Institut für Festkörperphysik, Technische Universität Berlin, Hardenbergstr. 36, 10623 \\ Berlin, Germany \\ ${ }^{b}$ Karlsruher Institut für Nanotechnologie, Hermann-von-Helmholtz-Platz 1, 76344 \\ Eggenstein-Leopoldshafen, Germany \\ ${ }^{c}$ Micro und Nanosysteme, Eidgenösische Technische Hochschule Zürich, Tannenstrasse \\ 3, 8092 Zürich, Switzerland \\ ${ }^{d}$ CNRS, Centre de Recherche Paul Pascal (CRPP), UPR 8641, 33600 Pessac, France \\ ${ }^{e}$ Applied Physical Chemistry, University of Heidelberg, Im Neuenheimer Feld 253, 69120 \\ Heidelberg, Germany \\ ${ }^{f}$ Joint Institute of Advanced Materials and Processes, Dr.-Mack-Strasse 81, 90762 Fürth, \\ Germany \\ ${ }^{g}$ Interdisciplinary Center for Molecular Materials, Friedrich Alexander Universität \\ Erlangen-Nürnberg, Henkestrasse 42, 91052 Erlangen, Germany
}

\section{Abstract}

Covalent functionalization of single-walled carbon nanotubes typically leads to an intensity increase of the defect-induced Raman mode ( $D$ mode). A large intensity ratio of the $D$ and $G$ modes $(D / G$ ratio) is therefore often used as evidence for a successful functionalization. Here, we discuss the effect of the $D$-mode resonance on the $D / G$ ratio and compare pristine and covalently functionalized nanotubes. By resonance Raman spectroscopy we study the evolution of the lineshape and frequencies of the $D$ and $2 D$ modes of samples enriched with semiconducting and metallic nanotubes in comparison with the radial breathing mode. First, we experimentally demonstrate

\footnotetext{
* Corresponding author

Email address: janina.maultzsch@physik.tu-berlin.de (Janina Maultzsch)
} 
the dependence of the $D$ - and $2 D$-mode frequencies on the tube diameter and derive an analytical expression for both frequencies as a function of the diameter and the optical transition. Furthermore, we demonstrate that the contribution of the $D$ and $2 D$ modes for small SWCNTs disappears in covalently functionalized samples due to partial destruction. In fact, this can change the measured $D / G$ ratio significantly and has a fundamental impact on the determination of the degree of SWCNT functionalization by Raman spectroscopy.

Keywords: Carbon nanotubes, Raman spectroscopy, defects, functionalization, diameter dependence

\section{Introduction}

The intrinsic properties of single-walled carbon nanotubes (SWCNTs) [1, 2, 3] make them an ideal candidate for applications like sensors [4, 5, 6, 7, 8, 9, 10, electronic devices [11, 12, 13, 14, 15, 16], or catalysts [17, 18, 19].

5 In many cases, applications require a functionalization of the SWCNTs to account for application-specific properties. In addition, the covalent functionalization of SWCNTs can enhance their debundling and increase their solubility [20, 21]. Moreover, electronic type specific functionalization leads to an enrichement of semiconducting or metallic species and thus presents itself as a good alternative for the use of surfactants [22, 23, 24, 25, 26].

For monitoring the process of covalent functionalization, the intensity increase of the defect-induced Raman mode ( $D$ mode) is the most frequently used indicator. Typically, the degree of functionalization is determined by the amplitude or intensity ratio between the $D$ mode and the $G$ mode [27, 28] ( $D / G$ ratio). As recently shown, for an excitation in resonance with the optical transitions, the $D$-mode intensity is strongly enhanced [29]. A still open question is how the resonance behavior of the $D$ mode influences the $D / G$ ratio in SWCNTs ensembles, typically used for applications. Associated with this question is the possibility to monitor a selective covalent function- 
alization of nanotubes by using excitation energies with semiconducting or metallic tubes in resonance.

In this work, we attribute the $D$ - and $2 D$-mode (in the following referred to as (2) $D$ mode) contributions of samples enriched with semiconducting and metallic nanotubes [22] to different optical transitions and determine the diameter dependence of the (2) $D$-mode frequency. To support our results, we compare our determined diameter dependence with measurements of samples with well known diameter, like nanotube samples highly enriched with single chiralities [30] and measurements of individual, freestanding SWCNTs [31]. Furthermore, we analyze the influence of the $D$-mode diameter dependence on the $D / G$ ratio of functionalized tubes and the possibility to monitor an electronic type selective functionalization.

\section{Experimental details and sample preparation}

Raman measurements were performed with a microscope setup in backscattering geometry. A titanium-sapphire $(1.49-1.77 \mathrm{eV})$, a dye laser $(1.79-$ $2.05 \mathrm{eV}$ ), and several fixed laser lines were used for excitation with $1 \mathrm{~mW}$ laser power. All spectra were calibrated using a neon lamp. The samples enriched with metallic and semiconducting tubes [sample set (i)] were produced by size-exclusion chromatography with SWCNTs obtained from Pulsed Laser Vaporization (PLV) 22]; the samples enriched with single chiral indices [samples (ii)] were produced by ultra centrifugation (DGU) of CoMoCAT- and PLV-produced nanotubes for the $(7,5)$ and $(9,7)$ SWCNT samples, respectively [30]. The individual, freestanding nanotubes [samples (iii)] were grown by catalyzed chemical vapor deposition [31]. The used catalyst particles had been adsorbed on $3 \mu \mathrm{m}$ thick thermally oxidized polycrystalline Si support, patterned as sharp opposing tips [32]. Covalent sidewall-functionalized HiPco SWCNTs bearing carboxyl groups [samples (iv)] were prepared by reductive exfoliation/functionalization under modified Birch conditions with subsequent separation by DGU to remove unfunctionalized and bundled carbon 


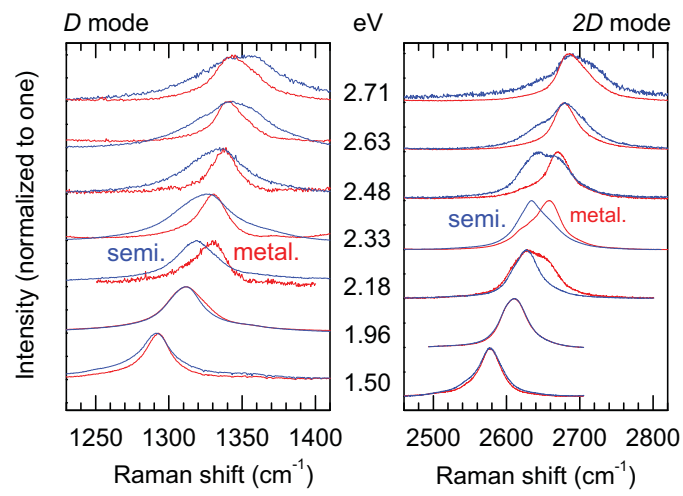

Figure 1: Raman spectra of the $D$ mode (left) and the $2 D$ mode (right) of sample SSWCNT (blue) and M-SWCNT (red), for excitation energies between 1.5 and $2.71 \mathrm{eV}$ (bottom to top). Intensities are normalized to one; spectra are vertically offset for clarity.

nanotubes [33, 34]. The spectra were collected from nanotubes in solution drop-casted on $\mathrm{Si} / \mathrm{SiO}_{2}$ or directly from aqueous solution.

\section{Results and discussion}

In the following, we will analyze different sets of nanotube samples, which are explained in detail in the experimental section above. The sample sets are: (i) samples enriched with semiconducting and metallic carbon nanotubes, which will be referred to as S-SWCNT and M-SWCNT, respectively; (ii) SWCNT samples enriched with single chiral indices, namely $(9,7)$ and $(7,5)$ SWCNTs; (iii) individual SWCNTs in the diameter range of approximately $1.5 \mathrm{~nm}$; (iv) covalently sidewall-functionalized SWCNTs.

Figure 1 shows the $D$ and $2 D$ Raman modes of samples enriched with semiconducting (S-SWCNT, blue) and metallic (M-SWCNT, red) nanotubes for different excitation energies. The amplitudes are normalized to one. Above $1.96 \mathrm{eV}$ excitation energy, the lineshapes of the (2)D mode are significantly different for the two samples. By comparing this observation to the experimental Kataura plot of the RBM [36, 37] (Fig. 2), we observe that above $1.96 \mathrm{eV}$ the second semiconducting optical transition $\left(E_{22}^{S}\right)$ and addi- 


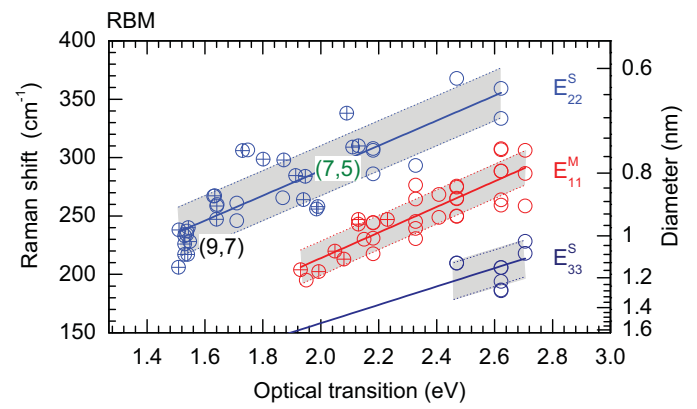

Figure 2: Experimental Kataura plot of the sample S-SWCNT and M-SWCNT, ordered by optical transitions. Crossed symbols denote transition energies gained from resonance profiles of the RBM, open symbols denote transition energies gained from incomplete resonance profiles or discrete excitation energies. Solid lines represent linear fits of the different optical transitions with indication of the standard deviations (light-gray areas). For the $E_{33}^{S}$ transition, we introduced an additional fit point at $(0,0)$. Specific data points from $(9,7)$ and $(7,5)$ nanotubes are indicated.

tionally the first metallic optical transition $\left(E_{11}^{M}\right)$ of the nanotubes become resonant. Above this energy, an additional contribution to the (2) $D$ mode with a slightly higher frequency is observed; most prominent for the sample M-SWCNT (Fig. 1). Above $2.48 \mathrm{eV}$, even the third optical transition of semiconducting nanotubes $\left(E_{33}^{S}\right)$ becomes resonant (Fig. 2); again a new contribution appears in the Raman spectra of the (2)D mode with an even higher frequency; most prominent for the sample S-SWCNT (Fig. 1, best visible for the $2 D$ mode).

In Fig. 3 we analyze the peak dispersions with laser excitation energies of the different contributions in the (2) $D$ mode, by phenomenologically fitting the spectra with up to three Lorentzians for the $D$ mode and with up to four Lorentzians for the $2 D$ mode 1 . The sum of all areas was normalized to one for each spectrum. The areas of the symbols in Fig. 3 indicate the relative intensities of the Lorentzians; the shape denotes the different sam-

\footnotetext{
${ }^{1}$ We found that the contribution of the $2 D$ mode related to the optical transition $E_{22}^{S}$ consists of two Lorentzians [29]. This leads to up to four contributions for the $2 D$ mode, involving only three optical transitions.
} 


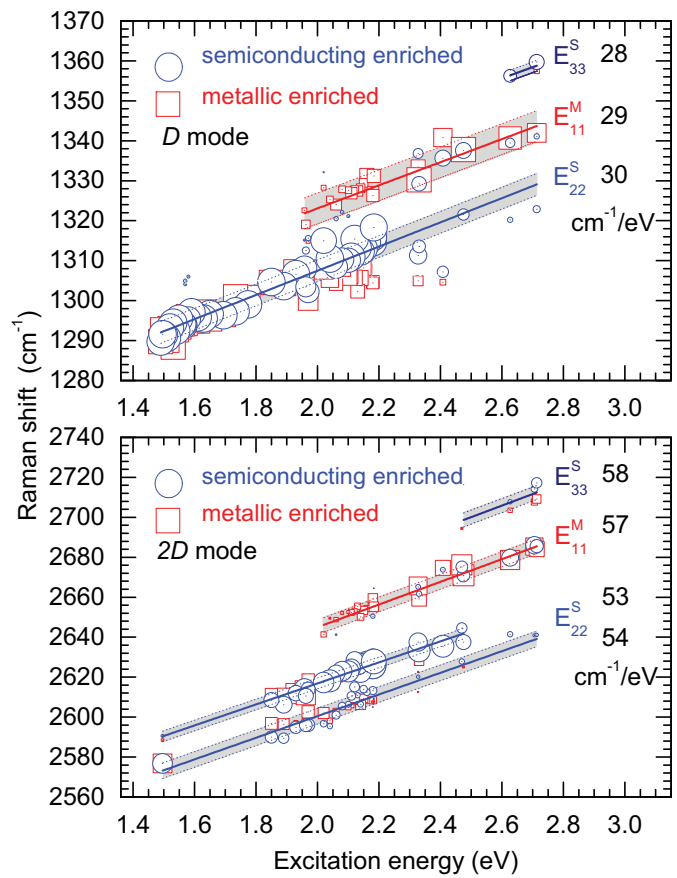

Figure 3: Lorentzian fits of the different contributions of the $D$ mode (upper panel) and the $2 D$ mode (lower panel) for the sample S-SWCNT (blue circle) and M-SWCNT (red square). The areas of the symbols indicate the relative intensities of the Loreantzian fits. The solid lines are the linear fits of the different contributions with indication of the standard deviations (light-gray areas). The transition $E_{22}^{S}$ shows two contributions for the $2 D$ mode [35, 29]. The optical transitions and the values for the dispersion (frequency shift vs. excitation energy) are denoted. 
ples, i.e., metallic or semiconducting enriched CNTs. From the comparison of the relative intensities, we find for the $D$ mode that there are certain resonance windows with mainly semiconducting $(\approx 1.5-2.0 \mathrm{eV})$ or metallic tubes $(\approx 2.4-2.6 \mathrm{eV})$ in resonance. Further, each contribution shows a similar dispersion with a mean value of $\approx 29 \pm 5 \mathrm{~cm}^{-1} / \mathrm{eV}$ for the $D$ mode and $\approx 56 \pm 6 \mathrm{~cm}^{-1} / \mathrm{eV}$ for the $2 D$ mode. These dispersion values are much smaller compared to single-tube measurements [29] or compared to measurements on not enriched nanotube ensembles [38. The values shown in Fig. 3 reflect the diameter dependence of the (2)D mode in a SWCNT ensemble for subsequently resonantly excited nanotubes at a given optical transition, as discussed below.

Due to the similar energy for incoming resonance for the RBM and (2) $D$ mode [29] it seems reasonable to deduce the dependence of the (2) $D$-mode frequency on diameter from a direct comparison with the diameter of the related RBM. With $\omega_{\mathrm{RBM}}$ proportional to $1 / d$ [39] and by neglecting the chiral-angle dependence of the optical transitions (branches), $E_{i i}$ is also roughly proportional to $1 / d$. It follows that $\omega_{\mathrm{RBM}}$ is approximately proportional to $E_{i i}$. Therefore, each optical transitions of the RBM Kataura plot can be linearly fitted (Fig. 2), which assigns a certain excitation energy to an RBM frequency. Figure 2 shows these linear fits for the experimental Kataura plot of sample S-SWCNT and M-SWCNT in the diameter range of $0.6-1.2 \mathrm{~nm}$. Because of neglecting the chiral-angle dependence of the optical transitions (branches), this approximation will lead only to a mean value for the diameter dependence of the (2)D mode in SWCNT ensembles. The variance due to the different branches of the optical transitions is approximated by the standard deviations $\sigma_{\text {RBM }}$ of the fits (Fig. 2, gray areas). Furthermore, only the incoming resonance is taken into account.

The linear equations for the fit of the RBM and the $D$ and $2 D$ modes are 
given by:

$$
\begin{aligned}
& \omega_{\mathrm{RBM}}\left(E_{i i}\right)=m_{\mathrm{RBM}} \cdot E_{i i}+b_{\mathrm{RBM}} \pm \sigma_{\mathrm{RBM}} \\
& \omega_{(2) D}\left(E_{i i}\right)=m_{(2) D} \cdot E_{i i}+b_{(2) D} \pm \sigma_{(2) D}
\end{aligned}
$$

with slope $m$, intercept $b$ and standard deviation $\sigma$. Equations $1 \mathrm{a}$ and $1 \mathrm{~b}$ are solved for the phonon frequency $\omega_{R B M}\left(\omega_{(2) D}\right)$ which gives

$$
\omega_{\mathrm{RBM}}\left(\omega_{(2) D}\right)=m^{*} \cdot \omega_{(2) D}\left(E_{i i}\right)+b^{*}
$$

with a new slope $m^{*}$ and intercept $b^{*}$ :

$$
\begin{gathered}
m^{*}=\frac{m_{\mathrm{RBM}}}{m_{(2) D}} \\
b^{*}=b_{\mathrm{RBM}} \pm \sigma_{\mathrm{RBM}}-\frac{m_{\mathrm{RBM}}}{m_{(2) D}}\left(b_{(2) D} \pm \sigma_{(2) D}\right)
\end{gathered}
$$

Using Eq. (2) and the diameter dependence of the RBM [37], the dependence of the diameter on $\omega_{(2) D}$ can be calculated:

$$
d\left(\omega_{(2) D}\right)=\frac{C_{1}}{m^{*} \cdot \omega_{(2) D}\left(E_{i i}\right)+b^{*}-C_{2}} .
$$

The parameters $C_{1}$ and $C_{2}$ have been determined theoretically and experimentally in various ways [39]. In this work we will use the parameters experimentally obtained by resonance Raman spectroscopy [37] with $C_{1}=$ ${ }_{110} 215 \mathrm{~nm} \cdot \mathrm{cm}^{-1}$ and $C_{2}=18 \mathrm{~cm}^{-1}$ for HiPCO tubes in solution with a similar diameter range as in the present sample. The parameters for the linear fits of RBM and (2)D mode are summarized in Tab. 1 .

In the following section, we compare our estimated diameter dependence from Eq. (5) with measurements of SWCNTs exhibiting well-defined diame115 ters, like samples enriched with single chiral indices [sample $(9,7)$ and $(7,5)$ ] 
Table 1: Fit parameters of the linear fits related to the optical transitions of the RBM and the (2) $D$ mode; slope $m$, intercept $b$, and standard deviation $\sigma$ are given.

\begin{tabular}{ccccc}
\hline \hline Fit & \multicolumn{4}{c}{ Optical transition } \\
parameters & $E_{22}^{S}$ & $E_{22}^{\prime}$ & $E_{11}^{M}$ & $E_{33}^{S}$ \\
\hline$m_{\mathrm{RBM}}$ & 107.0 & & 109.6 & 78.5 \\
$b_{\mathrm{RBM}}$ & 74.9 & & -5.3 & 1.1 \\
$\sigma_{\mathrm{RBM}}$ & 21.4 & & 14.7 & 15.7 \\
\hline$m_{D}$ & 30.3 & & 28.9 & 27.9 \\
$b_{D}$ & 1246.9 & & 1265.3 & 1283.0 \\
$\sigma_{D}$ & 2.7 & & 3.9 & 1.3 \\
\hline$m_{2 D}$ & 54.2 & 52.6 & 56.8 & 57.7 \\
$b_{2 D}$ & 2492.1 & 2511.6 & 2531.5 & 2555.8 \\
$\sigma_{2 D}$ & 3.9 & 2.6 & 3.7 & 3.5 \\
\hline \hline
\end{tabular}

and individual, freestanding SWCNTs. The results are shown in Figure 4 (a) and confirm our calculated diameter dependence in an excellent way. The sample $(9,7)$ and $(7,5)$ were measured using a broad range of excitation energies 29] (denoted with line segments in the plot). The radii of the circles indicate the measured intensities, which were normalized to the maximum $D$ mode intensity recorded within the measurement range for each sample. As can be seen, the $D$-mode frequencies with the highest intensities match the linear fit for the optical transition $E_{22}^{S}$ very well. This good agreement can be understood from the fact that the samples S-SWCNT and M-SWCNT also contain $(9,7)$ and $(7,5)$ tubes (compare Fig. 2). In contrast to the resonance Raman profiles of the $(9,7)$ and $(7,5)$ CNTs, the individual SWCNTs (blue, open circles) were measured using a single excitation energy of $2.33 \mathrm{eV}$. As can be seen, the $D$-mode frequencies of these nanotubes do not match with the frequencies measured for sample S-SWCNT and M-SWCNT [Fig. 4(a), light-gray areas]. However, by extending the linear fit of the optical transition $E_{33}^{S}$ to lower frequencies (dashed line), we observe a fairly good agreement with our experimental data and the calculated $D$-mode frequency. The opti- 

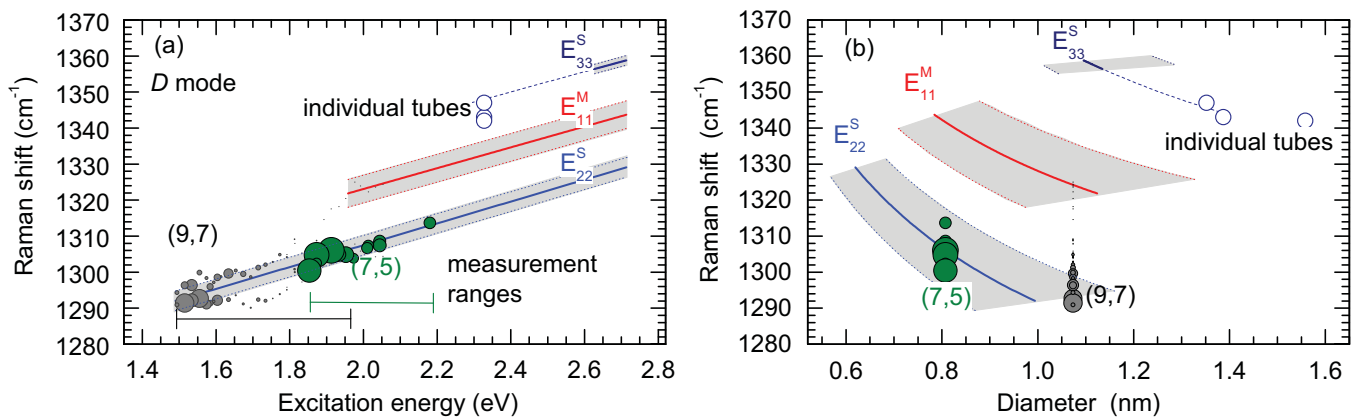

Figure 4: (a) Linear fits of the measured $D$-mode dispersions from Fig. 3 (lines) with standard deviations (gray areas) in comparison to samples with well-defined diameter (circles). (b) Calculated dependence of the $D$-mode frequency on diameter from Eq. (5) for the different optical transitions $E_{i i}$ (lines) with standard deviations (light-gray areas) in comparison to samples with well-defined diameter (circles).

cal transition energies of the individual SWCNTs were determined from their RBM frequencies (not shown).

Figure 4(b) shows the calculated $D$-mode frequencies as a function of the diameter for the different optical transitions [Eq. (5)]. As can be seen, we observe an inversely-proportional relation between diameter and $D$-mode frequency for each optical transition. The solid lines denote the mean values and the light-gray areas indicate the standard deviations $\sigma$. The diameters of the sample $(9,7)$ and $(7,5)$ were directly calculated [39]; the diameter of the individual SWCNTs were determined from their RBM frequencies [37]. The results for the samples $(9,7)$ and $(7,5)$ match the calculated diameter dependence of the $E_{22}^{S}$ transition for the sample S-SWCNT and M-SWCNT (light-gray area). As could be already inferred from Fig. 4(a), the measured ${ }_{145} D$-mode frequencies of the individual SWCNTs, which correspond to the $E_{33}^{S}$ transition, do not match the calculated diameter dependence directly. Again, by extending the diameter range of our theoretical model for the $E_{33}^{S}$ transition to larger diameters, we observe a good agreement between experiment and theory. This can be understood from the fact that the individual CNTs exhibit larger diameters compared to samples S-SWCNT and M-SWCNT and therefore lower $D$-mode frequencies [compare Fig. 4 (a)]. 


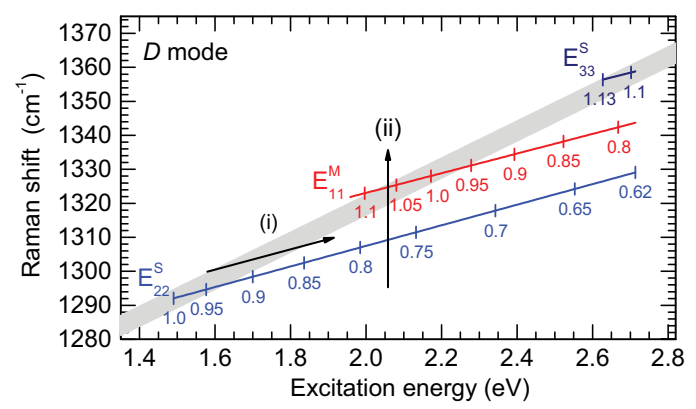

Figure 5: Linear fits of the measured $D$-mode dispersions from Fig. 3 (lines) with denotation of the calculated diameters (nm) from Eq. (5). Arrows (i) and (ii) indicate different relations between Raman frequency and diameter for different experiments (see text). Thick, gray line is a linear fit through the phonon frequencies related to nanotubes with a diameter of $1 \mathrm{~nm}$.

Figure 5 shows the correlation between the $D$-mode dispersion and the tube diameter in a single plot. For each optical transition the calculated diameters are denoted (in units of nm). From this picture, we see that it is possible to obtain two contrary relations between Raman frequency and diameter:

(i) On the one hand, if we tune the excitation energy and follow the $D$-mode frequencies along only one optical transition in a nanotube ensemble (as indicated here for $E_{22}^{S}$ ), we find an inversely-proportional diameter dependence [e.g. the $D$-mode frequencies for sample $(9,7)$ and $(7,5)]$.

(ii) On the other hand, if we perform measurements with a fixed excitation energy on nanotube samples with different mean diameters, there will be different optical transitions in resonance (as indicated here for $E_{22}^{S}$ and $E_{11}^{M}$ ), and the $D$-mode frequencies will be proportional to their diameter.

A simulation of the (2) $D$-mode diameter dependence in nanotube ensembles and a description of the curvature induced jump between the optical transitions can be found in Ref. [40].

Figure 6 shows a theoretical Kataura plot for the $D$ mode from Eq. (5) (solid lines, thick segments denote the range of measurement) in comparison with results from literature. Symbols are taken from literature, with 


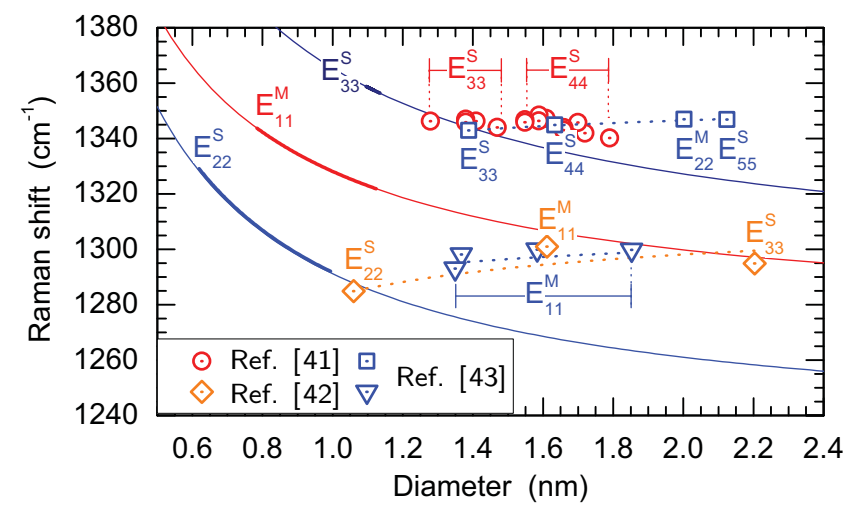

Figure 6: Comparison between calculated diameter dependence of the $D$ mode (solid lines) from Fig. 4 (b) and literature. The red circles (Ref. [41]) are data from measurements following the optical transition $E_{33}^{S}$ and $E_{44}^{S}$. The orange diamonds (Ref. [42]), blue triangles, and blue squares (Ref. [43, different symbols indicate different excitation energies) are data from measurements for different optical transitions.

their assigned optical transitions denoted next to them. The red circles are measurements from Ref. [41] following the $E_{33}^{S}$ and the $E_{44}^{S}$ transitions. The results for $E_{33}^{S}$ match our calculations very well and show the predicted inversely-proportional diameter dependence following one optical transition ( $E_{44}^{S}$ shows the same behavior). The blue triangles from Ref. [43] were assigned all to the optical transition $E_{11}^{M}$, but only some of them fit to our calculated diameter dependence. The reason is that in Ref. [43] a splitting of the $D$ mode was not taken into account and therefore, the data points at $\approx 1.4 \mathrm{~nm}$ are in fact a superposition of the optical transitions $E_{22}^{S}$ and $E_{11}^{M}$. The blue squares (Ref. [43]) and the orange diamonds (Ref. [42]) are measurements probing different optical transitions and were erroneously fitted in literature with $\omega_{D}=C_{1}-\left(C_{2} / d\right)$ (dashed lines). However, as can be seen from Fig. 6, the $D$-mode frequency increases with increasing nanotube diameter, in nice agreement with our model [compare Fig. 5. arrow (ii)]. The orange diamond at $\approx 2.2 \mathrm{~nm}$ was assigned in Ref. [42] to $E_{33}^{S}$, but seems rather to belong to $E_{11}^{M}$. For the $2 D$ mode we find the same good agreement with Ref. [44] (not shown). 

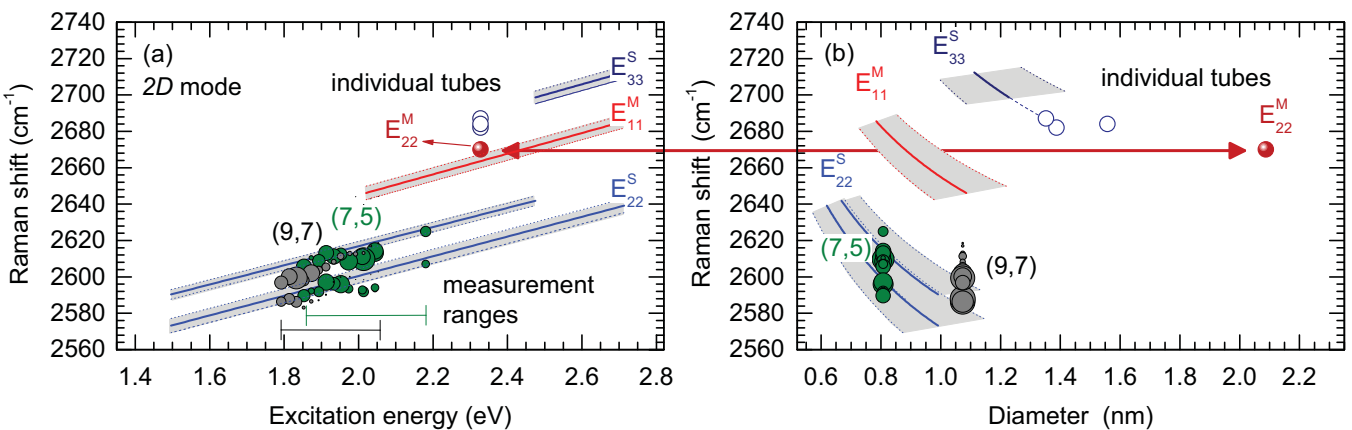

Figure 7: Measured 2D-mode dispersion (a) and calculated diameter dependence (b) of the $2 D$ mode for sample S-SWCNT and M-SWCNT with comparisons to samples with well-defined diameter.

Based on our diameter assignment, the earlier reported dispersions of $\approx$ $50 \mathrm{~cm}^{-1} / \mathrm{eV}\left(100 \mathrm{~cm}^{-1} / \mathrm{eV}\right)$ for the $D$ mode (2D mode) in SWCNT ensembles [45, 46, 47] can be understood as a superposition of different contributions of different optical transitions. For nanotubes produced with the HiPCO process, a mean diameter of $\approx 1 \mathrm{~nm}$ can be assumed [48]. Figure 5 shows a linear fit through the $D$-mode frequencies related to nanotubes with a diameter of $1 \mathrm{~nm}$, involving all optical transitions (thick, gray line). We find a dispersion of $55 \mathrm{~cm}^{-1} / \mathrm{eV}$, which is in good agreement with literature values from SWCNT ensembles. Furthermore, different mean diameters for nanotube ensembles will lead to variations in the dispersions.

Figure 7 shows the corresponding analysis of the diameter dependence for the $2 D$ mode; in general, we observe the same behavior as for the $D$ mode 2 . A special feature is the measurement of an individual metallic SWCNTs, in resonance with the second metallic optical transition $E_{22}^{M}$ (filled, red circle). The measured Raman frequency is located in the area assigned to the $E_{11}^{M}$ transition [Fig. 7(a)]. This nanotube possesses a much larger diameter than the tubes in samples S-SWCNT and M-SWCNT, as can be seen in Fig. 7(b).

\footnotetext{
${ }^{2}$ The strongest intensity for sample $(9,7)$ has to be assumed around $1.5 \mathrm{eV}$ and could not be measured due to the limited energy range in our measurements [Fig. 77.(a)].
} 
This shows that nanotubes with a large difference in diameter can have the same (2) $D$-mode frequency if excited at different optical transitions [depicted by the red arrow linking Fig. 7(a) and (b)]. Therefore, the determined dependence of the (2) $D$-mode frequency given by Eq. (5) is only clear for SWCNT ensembles containing tubes in a small diameter range. For SWCNTs produced in the HiPCO process [48], this range is between $\approx 0.6-1.2 \mathrm{~nm}$. For samples containing nanotubes with a large diameter range, different optical transitions overlap in frequency, and the dependence on diameter will be ambiguous.

The diameter dependence of the $D$ mode makes it possible to correlate the often observed functionalization-induced decrease in RBM intensity to the related metallic and semiconducting contributions of the $D$ mode. For instance, an excitation energy of $1.7 \mathrm{eV}$ will probe the $D$ mode of semiconducting nanotubes with a mean diameter of $0.9 \mathrm{~nm}$ (Fig. 5). A decrease in RBM intensity can be due to two reasons: First, a functionalization process changes the $s p^{2}$ hybridized carbon-scaffold of the SWCNTs, which leads to a decrease in intensity of Raman modes [49]. The decrease in RBM intensity is therefore often used as a proof of selective functionalization, related to diameter [50, 51] and species [52, 53, 33, 26]. Second, a very high degree of functionalization can alter the $s p^{2}$ hybridized carbon-scaffold to such an extent that the tube will be completely destroyed. This also leads to a decrease in Raman intensity. To distinguish between these two effects, the assignment of the $D$ mode to diameter and species can be used. The contributions of the $D$ mode will increase for functionalized SWCNTs and disappear for destroyed SWCNTs.

Figure 8 shows the Raman spectra of the RBM, $D, G$ and $2 D$ mode for covalently sidewall-functionalized SWCNTs 33, (red) and the reference material (black) for two excitation energies; normalized to acquisition power and time. For both excitation energies, the RBMs at high frequencies disappear after the functionalization process [Fig. 8(a)]. For the shown excitation 


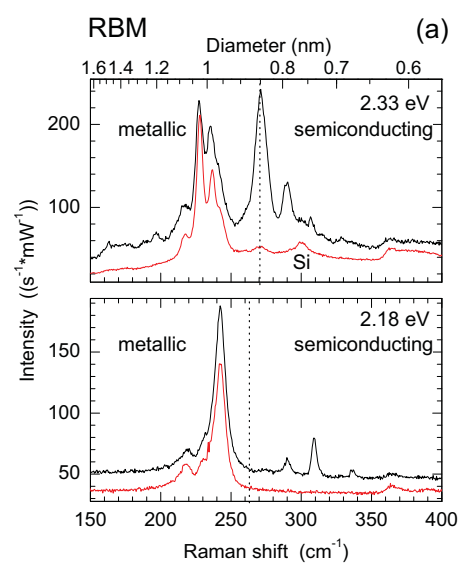

(a) $\quad D$ and $G$ mode

(b)

$2 D$ mode

(c)
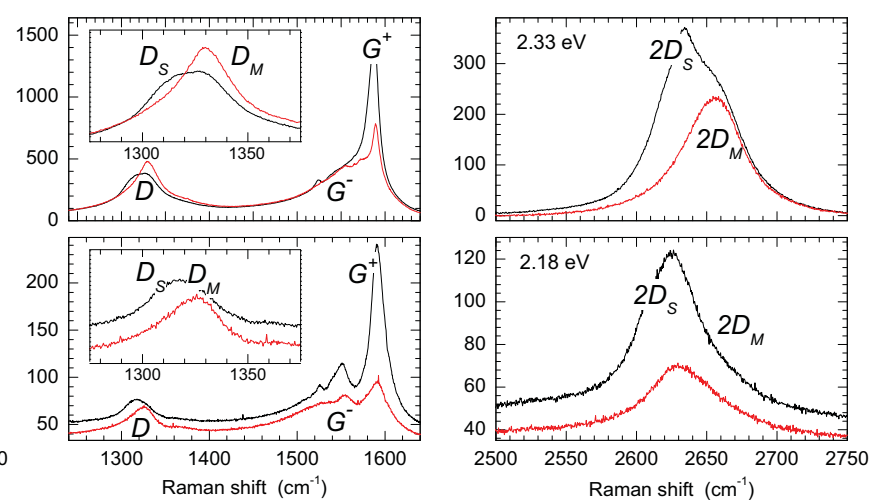

Figure 8: Raman spectra of functionalized SWCNTs (red) and reference (black) for 2.33 (upper panel) and $2.18 \mathrm{eV}$ excitation (lower panel); intensity normalized to acquisition power and time. (a) RBM with assignment to semiconducting and metallic tubes, (b) $D$ and $G$ mode, inset shows zoom to the $D$ mode, (c) $2 D$ mode.

energies, these RBMs belong to small $(0.7-0.8 \mathrm{~nm})$ semiconducting SWCNTs [37]. The contribution of the $D$ mode related to these nanotubes has a Raman frequency in the range of $1290-1320 \mathrm{~cm}^{-1}$ [Fig. 4(b)]. A decrease of the RBM due to functionalisation would lead to an increase of this semiconducting-tube related contribution of the $D$ mode $\left(D_{S}\right)$. But as we see in the inset of Fig. 8(b), $D_{S}$ decreases at both excitation energies. The same intensity decrease is observed in the $2 D$ mode $\left[2 D_{S}\right.$, Fig. 8(c)]. This indicates that harsh functionalization conditions have destroyed the small nanotubes in this sample. Furthermore, the metallic contribution of the $D$ mode $\left(D_{M}\right)$ remains constant [just like the RBM intensities related to metallic SWCNTs, Fig. 8 (a)] or is even slightly increased.

Since the degree of functionalization is commonly deduced from the intensity ratio between the $D$ and $G$ mode [28] ( $D / G$ ratio), it is also important to analyze possible changes in the $G$ mode caused by the functionlization process. In Fig. 8(b), we see that the broad $G^{-}$mode, which is related to metallic SWCNTs [54, 55, 47, 56], is almost unchanged in intensity, whereas 
the $G^{+}$mode is much weaker in the functionalized sample. This intensity decrease can be identified with the destruction of the small, semiconducting SWCNTs [56]; similar observations were made by Refs. [57] and [58] before. As a consequence, the nearly unchanged $D_{M}$ mode in relation to the strongly decreased $G^{+}$mode leads to an overestimated $D / G$ ratio. Thus, a quite high degree of functionalization would be deduced, however, the absolute intensity of the $D$ mode has only slightly changed [inset Fig. 8(b)].

To overcome this problem it is important to use different components of the $G$ mode for excitation energies with different species of nanotubes in resonance. For semiconducting tubes in resonance, the $G^{+}$mode is favorable. In contrast, for metallic tubes in resonance, the $G^{-}$mode should be considered. The use of the $G^{-}$mode can bear the problem that it might decrease due to functionalization [59, 60] caused by a shift in the Fermi level [61, 62]. To make the $D / G$-mode ratio comparable for different excitation energies it has to be normalized with the $D / G$ ratio of the reference material. Using this method, the normalized $D / G^{+}$ratio of the functionalized nanotubes increases by a factor of 2.8 for large-diameter, undestroyed semiconducting tubes in resonance $(1.96 \mathrm{eV})$ and for larger semiconducting tubes in resonance $(1.49 \mathrm{eV})$ even by the factor 8.2. For metallic tubes in resonance $(2.41 \mathrm{eV})$, the increase of the normalized $D / G^{-}$ratio is only a factor 1.4 . This behavior reflects the suspected preferred functionalization of semiconducting nanotubes in this sample [33].

\section{Conclusion}

In conclusion, we have shown that in SWCNT ensembles the (2) $D$ mode arises from a superposition of resonantly excited nanotubes. This leads to different contributions of the (2) $D$ mode, which can be assigned to different optical transitions and this directly leads to smaller dispersions in comparison to measurements of individual nanotubes. Furthermore, the resonance behavior of the (2)D mode makes it possible to assign these different contri- 
butions of the broad (2)D mode in a SWCNT ensemble to different diameters. This assignment predicts both, an inversely-proportional or a proportional dependence of the (2) $D$-mode frequency on the diameter, depending on the experimental conditions.

Furthermore, the resonance behavior of the $D$ mode can be used to prove selective functionalization. Changes in the $D / G$ ratio have to be analyzed carefully, especially for excitation energies with both, metallic and semiconducting tubes in resonance. A partial loss of the sample material can alter the $D / G$ ratio, caused by a decrease of the $G^{+}$-mode intensity and not caused by a successful functionalization. Therefore, to investigate a selective functionalization, the $G^{+}$mode has to be used to calculate the $D / G$ ratio with mainly semiconducting tubes contributing to the $D$ mode and the $G^{-}$for mainly metallic tubes contributing to the $D$ mode. For comparison of $D / G$ ratios at different excitation energies, a careful normalization to the reference material is crucial.

\section{Acknowledgement}

The authors thank Christofer Hierold (ETH Zürich) for useful discussions. This work was funded by the European Research Council (ERC) under grant no. 259286. We acknowledge further support by the ERC under grant no. 246622 "Graphenochem", and the DFG-funded SFB 953 Project A1, "synthetic carbon allotropes". M.M. and M.H. acknowledge financial support from the Swiss National Science Foundation (SNSF: 200020-121831) and financial support from the EU FP7 program Technotubes.

\section{References}

[1] J. Hone, M. Whitney, C. Piskoti, A. Zettl, Thermal conductivity of single-walled carbon nanotubes, Phys. Rev. B 59 (1999) R2514.

[2] P. G. Collins, P. Avouris, Nanotubes for electronics, Scientific American 283 (2000) 62-69. 
[3] D. Kim, L. Zhu, C.-S. Han, J.-H. Kim, S. Baik, Raman characterization of thermal conduction in transparent carbon nanotube films, Langmuir 27 (2011) 14532-14538.

[4] J. J. Gooding, R. Wibowo, Liu, W. Yang, D. Losic, S. Orbons, F. J. Mearns, J. G. Shapter, D. B. Hibbert, Protein electrochemistry using aligned carbon nanotube arrays, J. Am. Chem. Soc. 125 (2003) 90069007.

[5] F. Patolsky, Y. Weizmann, I. Willner, Long-range electrical contacting of redox enzymes by swcnt connectors, Ang. Chem. 116 (2004) $2113-$ 2117.

[6] Y. W. Chang, J. S. Oh, S. H. Yoo, H. H. Choi, K.-H. Yoo, Electrically refreshable carbon-nanotube-based gas sensors, Nanotechnology 18 (2007) 435504 .

[7] C. Gao, Z. Guo, J.-H. Liu, X.-J. Huang, The new age of carbon nanotubes: An updated review of functionalized carbon nanotubes in electrochemical sensors, Nanoscale 4 (2012) 1948-1963. doi:10.1039/ C2NR11757F.

[8] A. Boyd, I. Dube, G. Fedorov, M. Paranjape, P. Barbara, Gas sensing mechanism of carbon nanotubes: From single tubes to high-density networks, Carbon 69 (2014) 417 - 423. doi:http://dx.doi.org/10. 1016/j.carbon.2013.12.044.

[9] T. Helbling, C. Roman, C. Hierold, Signal-to-noise ratio in carbon nan330 otube electromechanical piezoresistive sensors, Nano Letters 10 (2010) 3350-3354. doi:10.1021/nl101031e.

[10] K. Chikkadi, M. Muoth, V. Maiwald, C. Roman, C. Hierold, Ultra-low power operation of self-heated, suspended carbon nanotube gas sen- 
sors, Applied Physics Letters 103 (22) (2013) 223109. doi:10.1063/1. 4836415.

[11] M. Shim, A. Javey, N. W. Shi Kam, H. Dai, Polymer functionalization for air-stable n-type carbon nanotube field-effect transistors, J. Am. Chem. Soc. 123 (2001) 11512-11513.

[12] A. Star, J.-C. P. Gabriel, K. Bradley, G. Gruner, Electronic detection of 340 specific protein binding using nanotube fet devices, Nano Lett. 3 (2003) 459-463.

[13] Z. Wu, Z. Chen, X. Du, J. M. Logan, J. Sippel, M. Nikolou, K. Kamaras, J. R. Reynolds, D. B. Tanner, A. F. Hebard, A. G. Rinzler, Transparent, conductive carbon nanotube films, Science 305 (2004) 1273-1276.

[14] G. Gruner, Carbon nanotube films for transparent and plastic electronics, J. Mater. Chem. 16 (2006) 3533-3539.

[15] M. M. Shulaker, G. Hills, N. Patil, H. Wei, H.-Y. Chen, H.-S. P. Wong, S. Mitra, Carbon nanotube computer, Nature 501 (2013) 526-530.

[16] M. F. L. De Volder, S. H. Tawfick, R. H. Baughman, A. J. Hart, Carbon nanotubes: Present and future commercial applications, Science 339 (2013) 535-539. doi:10.1126/science.1222453.

[17] P. Serp, M. Corrias, P. Kalck, Carbon nanotubes and nanofibers in catalysis, Appl. Cat. A: Gen. 253 (2003) 337-358.

[18] X. Pan, Z. Fan, W. Chen, Y. Ding, H. Luo, X. Bao, Enhanced ethanol production inside carbon-nanotube reactors containing catalytic particles, Nature materials 6 (2007) 507-511.

[19] J. Zhang, X. Liu, R. Blume, A. Zhang, R. Schlögl, D. Su, Surfacemodified carbon nanotubes catalyze oxidative dehydrogenation of nbutane, Science 322 (2008) 73. 
360

[20] A. Hirsch, O. Vostrowsky, Functionalization of carbon nanotubes, Topics in Current Chemistry 245 (2005) 193-238.

[21] B. Gebhardt, Z. Syrgiannis, C. Backes, R. Graupner, F. Hauke, A. Hirsch, Carbon nanotube sidewall functionalization with carbonyl compounds modified birch conditions vs the organometallic reduction approach, J. Am. Chem. Soc. 133 (2011) 7985-7995.

[22] K. Moshammer, F. Hennrich, M. Kappes, Selective suspension in aqueous sodium dodecyl sulfate according to electronic structure type allows simple separation of metallic from semiconducting single-walled carbon nanotubes, Nano Res. 2 (2009) 599-606.

[23] H. Liu, D. Nishide, T. Tanaka, H. Kataura, Large-scale single-chirality separation of single-wall carbon nanotubes by simple gel chromatography, Nature comm. 2 (2011) 309.

[24] F. Hof, S. Bosch, J. M. Englert, F. Hauke, A. Hirsch, Statistical raman spectroscopy: A method for the characterization of covalently functionalized single-walled carbon nanotubes, Angewandte Chemie International Edition 51 (2012) 11727-11730. doi:10.1002/anie.201204791.

[25] S. Bosch, L. Zeininger, F. Hauke, A. Hirsch, A supramolecular approach for the facile solubilization and separation of covalently functionalized single-walled carbon nanotubes, Chemistry - A European Journal 20 (2014) 2537-2541. doi:10.1002/chem.201303506.

[26] J. Gebhardt, S. Bosch, F. Hof, F. Hauke, A. Hirsch, A. Gorling, Selective reduction of swcnts - concepts and insights, J. Mater. Chem. C (2016) doi:10.1039/C5TC01407G.

[27] R. Graupner, Raman spectroscopy of covalently functionalized singlewall carbon nanotubes, J. Raman Spectrosc. 38 (2007) 673-683. 
[28] Y. Maeda, K. Saito, N. Akamatsu, Y. Chiba, S. Ohno, Y. Okui, M. Yamada, T. Hasegawa, M. Kako, T. Akasaka, Analysis of functionalization degree of single-walled carbon nanotubes having various substituents, J. Am. Chem. Soc. 134 (2012) 18101-18108.

390

395

[31] M. Muoth, T. Helbling, L. Durrer, S.-W. Lee, C. Roman, C. Hierold, Hysteresis-free operation of suspended carbon nanotube transistors, Nat. nanotech. 5 (2010) 589-592.

[32] M. Muoth, F. Gramm, K. Asaka, L. Durrer, T. Helbling, C. Roman,

[29] J. Laudenbach, F. Hennrich, H. Telg, M. Kappes, J. Maultzsch, Resonance behavior of the defect-induced raman mode of single-chirality enriched carbon nanotubes, Phys. Rev. B 87 (2013) 165423.

[30] N. Stürzl, F. Hennrich, S. Lebedkin, M. M. Kappes, Near monochiral single-walled carbon nanotube dispersions in organic solvents, J. Phys. Chem. C 113 (33) (2009) 14628-14632. doi:10.1021/jp902788y.

S.-W. Lee, C. Hierold, Chirality assignment to carbon nanotubes integrated in mems by tilted-view transmission electron microscopy, Sens. Actuators B 154 (2011) 155-159.

[33] B. Gebhardt, F. Hof, C. Backes, M. Mueller, T. Plocke, J. Maultzsch, C. Thomsen, F. Hauke, A. Hirsch, Selective polycarboxylation of semiconducting single-walled carbon nanotubes by reductive sidewall functionalization, J. Am. Chem. Soc. 133 (2011) 19459-19473.

[34] C. Backes, Density gradient ultracentrifugation on carbon nanotubes according to structural integrity as a foundation for an absolute purity evaluation, Chem. Phys. Chem. 12 (2011) 2576-2580.

[35] J. Laudenbach, F. Hennrich, M. Kappes, J. Maultzsch, Resonance behavior of defect-induced modes in metallic and semiconducting singlewalled carbon nanotubes, phys. stat. sol. (b) 249 (12) (2012) 2460-2464. 
[36] H. Kataura, Y. Kumazawa, Y. Maniwa, I. Umezu, S. Suzuki, Y. Ohtsuka, Y. Achiba, Optical properties of single-wall carbon nanotubes, Synth. Met. 103 (1999) 2555-2558.

[37] J. Maultzsch, H. Telg, S. Reich, C. Thomsen, Radial breathing mode of single-walled carbon nanotubes: Optical transition energies and chiralindex assignment, Phys. Rev. B 72 (20) (2005) 205438.

[38] J. Maultzsch, S. Reich, C. Thomsen, Chirality-selective Raman scattering of the $D$ mode in carbon nanotubes, Phys. Rev. B 64 (2001) 121407.

[39] S. Reich, C. Thomsen, J. Maultzsch, Carbon Nanotubes: Basic Concepts and Physical Properties, Wiley-VCH, Weinheim, 2004.

[40] F. Herziger, A. Vierck, J. Laudenbach, J. Maultzsch, Understanding double-resonant raman scattering in chiral carbon nanotubes: Diameter and energy dependence of the $d$ mode, Phys. Rev. B 92 (2015) 235409. doi:10.1103/PhysRevB.92.235409.

[41] A. G. Souza Filho, A. Jorio, G. Dresselhaus, M. S. Dresselhaus, R. Saito, A. K. Swan, M. S. Ünlü, B. B. Goldberg, J. H. Hafner, C. M. Lieber, M. A. Pimenta, Effect of quantized electronic states on the dispersive raman features in individual single-wall carbon nanotubes, Phys. Rev. B 65 (2001) 035404.

[42] M. A. Pimenta, A. Jorio, S. D. M. Brown, A. G. Souza Filho, G. Dresselhaus, J. H. Hafner, C. M. Lieber, R. Saito, M. S. Dresselhaus, Diameter dependence of the Raman D-band in isolated single-wall carbon nanotubes, Phys. Rev. B 64 (2001) 41401.

[43] A. G. Souza Filho, A. Jorio, G. G. Samsonidze, G. Dresselhaus, M. A. Pimenta, M. S. Dresselhaus, A. K. Swan, M. S. Ünlü, B. B. Goldberg, R. Saito, Competing spring constant versus double resonance effects on 
the properties of dispersive modes in isolated single-wall carbon nanotubes, Phys. Rev. B 67 (2003) 035427.

[44] J. Cardenas, Diameter dependence of second-order raman features of graphene tubes, Chem. Phys. Lett. 430 (2006) 367-369.

[45] J. Kastner, T. Pichler, H. Kuzmany, S. Curran, W. Blau, D. Weldon, M. Delamesiere, S. Draper, H. Zandbergen, Resonance raman and infrared spectroscopy of carbon nanotubes, Chem. Phys. Lett. 221 (1994) $53-58$.

[46] C. Thomsen, Second-order raman spectra of single and multiwalled carbon nanotubes, Phys. Rev. B 61 (2000) 4542.

[47] S. D. M. Brown, P. Corio, A. Marucci, M. A. Pimenta, M. S. Dresselhaus, G. Dresselhaus, Second-order resonant raman spectra of single-walled carbon nanotubes, Phys. Rev. B 61 (2000) 7734.

[48] P. Nikolaev, M. J. Bronikowski, R. K. Bradley, F. Rohmund, D. T. Colbert, K. A. Smith, R. E. Smalley, Gas-phase catalytic growth of single-walled carbon nanotubes from carbon monoxide, Chem. Phys. Lett. 313 (1) (1999) 91-97.

[49] J. L. Bahr, J. Yang, D. V. Kosynkin, M. J. Bronikowski, R. E. Smalley, M. James, Functionalization of carbon nanotubes by electrochemical reduction of aryl diazonium salts: a bucky paper electrode, J. Am. Chem. Soc. 123 (2001) 6536-6542.

[50] Z. Chen, W. Thiel, A. Hirsch, Reactivity of the convex and concave surfaces of single walled carbon nanotubes (swcnts) towards addition reactions: Dependence on the carbon-atom pyramidalization, ChemPhysChem 4 (2002) 93-97. 
[51] S. Banerjee, S. S. Wong, Demonstration of diameter-selective reactivity

465

470 in the sidewall ozonation of swnts by resonance raman spectroscopy, Nano Lett. 4 (2004) 1445-1450.

[52] M. S. Strano, C. A. Dyke, M. L. Usrey, P. W. Barone, M. J. Allen, H. Shan, C. Kittrell, R. H. Hauge, J. M. Tour, R. E. Smalley, Electronic structure control of single-walled carbon nanotube functionalization, Science 301 (2003) 1519-1522.

[53] D. Wunderlich, F. Hauke, A. Hirsch, Preferred functionalization of metallic and small-diameter single walled carbon nanotubes via reductive alkylation, J. Mat. Chem. 18 (2008) 1493-1497.

[54] A. Kasuya, M. Sugano, T. Maeda, Y. Saito, K. Tohji, H. Takahashi, Y. Sasaki, M. Fukushima, Y. Nishina, C. Horie, Resonant raman scattering and the zone-folded electronic structure in single-wall nanotubes, Phys. Rev. B 57 (1998) 4999.

[55] M. A. Pimenta, A. Marucci, S. D. M. Brown, M. J. Matthews, A. M. Rao, P. C. Eklund, R. E. Smalley, G. Dresselhaus, M. S. Dresselhaus, Resonant raman effect in single-wall carbon nanotubes, J. Mater. Res 13 (1998) 2397.

[56] M. Fouquet, H. Telg, J. Maultzsch, Y. Wu, B. Chandra, J. Hone, T. F. Heinz, C. Thomsen, Longitudinal optical phonons in metallic and semiconducting carbon nanotubes, Phys. Rev. Lett. 102 (7) (2009) 75501.

[57] M. Yudasaka, M. Zhang, S. Iijima, Diameter-selective removal of single-wall carbon nanotubes through light-assisted oxidation, Chemical Physics Letters 374 (1-2) (2003) 132 - 136. doi:http://dx.doi. org/10.1016/S0009-2614(03)00686-9.

[58] Y. Miyata, Y. Maniwa, H. Kataura. 
490

[59] M. S. Strano, C. B. Huffman, V. C. Moore, M. J. O’Connell, E. H. Haroz, J. Hubbard, M. Miller, K. Rialon, C. Kittrell, S. Ramesh, R. H. Hauge, R. E. Smalley, Reversible, band-gap-selective protonation of single-walled carbon nanotubes in solution, J. Phys. Chem. B 107 (2003) 6979-6985.

495

[60] R. Graupner, J. Abraham, D. Wunderlich, A. Vencelová, P. Lauffer, J. Röhrl, M. Hundhausen, L. Ley, A. Hirsch, Nucleophilic-alkylationreoxidation: A functionalization sequence for single-wall carbon nanotubes, J. Am. Chem. Soc. 128 (2006) 6683-6689.

[61] B. Hatting, S. Heeg, K. Ataka, J. Heberle, F. Hennrich, M. M. Kappes, 500 R. Krupke, S. Reich, Fermi energy shift in deposited metallic nanotubes: A raman scattering study, Phys. Rev. B 87 (2013) 165442.

[62] Y. Wu, J. Maultzsch, E. Knoesel, B. Chandra, M. Huang, M. Y. Sfeir, L. E. Brus, J. Hone, T. F. Heinz, Variable electron-phonon coupling in isolated metallic carbon nanotubes observed by raman scattering, Phys.

505 Rev. Lett. 99 (2007) 027402. 